$10 \mid 2018$

Devenir écrivain

\title{
Le « devenir auctorial » d'Ahmadou Kourouma
}

\section{Patrick Corcoran}

URL : https://journals.openedition.org/coma/1158

DOI : 10.4000/coma. 1158

ISSN : 2275-1742

\section{Éditeur}

Institut des textes \& manuscrits modernes (ITEM)

\section{Référence électronique}

Patrick Corcoran, «Le « devenir auctorial » d'Ahmadou Kourouma », Continents manuscrits [En ligne], 10 | 2018, mis en ligne le 15 mars 2018, consulté le 12 janvier 2023. URL : http:// journals.openedition.org/coma/1158; DOI : https://doi.org/10.4000/coma.1158

Ce document a été généré automatiquement le 12 janvier 2023.

\section{cc) (i) (2)}

Creative Commons - Attribution - Pas d'Utilisation Commerciale - Pas de Modification 4.0 International - CC BY-NC-ND 4.0

https://creativecommons.org/licenses/by-nc-nd/4.0/ 


\title{
Le « devenir auctorial » d'Ahmadou Kourouma
}

\author{
Patrick Corcoran
}

\begin{abstract}
En Sciences humaines (le paradoxe), pressenti mais jamais analysé dans ses conséquences extérieures, est la présupposition et l'induction de toute réponse possible par la question même, et donc la vanité de l'analyse et de l'interprétation. Jacques Baudrillard, Les Stratégies fatales, Paris,

Grasset, 1983.
\end{abstract}

Parler du «devenir » d'un écrivain, de la genèse auctoriale, est nécessairement une affaire hasardeuse. La mise en parallèle, d'un côté, de l'élaboration d'une genèse textuelle, travail analytique opéré sur un dossier génétique, et, de l'autre, d'un commentaire sur les premiers pas du parcours souvent tâtonnant et semé d'embûches qui est celui de l'écrivain qui se cherche, se heurte très rapidement à des difficultés méthodologiques. La plus importante est de cerner précisément l'objet d'étude : quelles sont les traces dans la vie d'un écrivain qui méritent comparaison avec les plans, ébauches, premiers jets et brouillons de l'univers avant-textuel d'une œuvre littéraire, ce que Pierre-Marc de Biasi appelle «les restes, les scories, les débris induits par le travail de conception de l'œuvre ${ }^{1} »$ ? Comment constituer, pour un être humain, l'équivalent du dossier génétique, cet objet matériel infiniment provisoire qui est déployé pour capter le processus même d'un devenir tout en différant la volonté de clôturer la démarche par une interprétation fixe? Il serait sans doute tentant de considérer le profil psychologique de l'individu, à la fois forgé et se forgeant dans un contexte culturel, politique, sociologique et historique déterminé, comme un élémentclé de ce "dossier" putatif. La mise-en-fiction de la vie de l'auteur servirait ainsi comme explication adéquate de la réalisation a posteriori de ses ambitions. Un cercle se referme dans ce genre de raisonnement. Il semble destiné à passer à côté de toute ouverture sur un réel processus de devenir. Une argumentation inductive de ce genre permet certes de dresser un portrait de l'écrivain en herbe, d'identifier les obstacles 
qu'il/elle a dû surmonter et les facteurs qui ont pu faciliter la réalisation de ses ambitions. Cependant, ni le contexte dans lequel l'individu évolue, ni la puissance de son désir de « devenir écrivain » ne suffisent à expliquer ce qui a pu faire la différence entre l'échec ou la réussite éventuelle de ses ambitions. Le résultat n'est en rien garanti par les préambules. Donc, une biographie présentée sous cette forme «aboutie » de l'ambition réalisée n'éclaire malheureusement qu'une partie d'une genèse auctoriale, et il est fort douteux qu'elle puisse prétendre à expliquer quoi que soit.

2 Considéré à la lumière de ces premières remarques, le cas d'Ahmadou Kourouma est instructif. Son émergence comme écrivain correspond à un moment crucial de sa vie et de celle de la Côte d'Ivoire. L'histoire complexe de l'écriture et de l'édition du premier roman de Kourouma, Les Soleils des indépendances, se prête donc facilement à une lecture biographique. À son retour dans son pays natal nouvellement indépendant, Kourouma se trouve happé par une conjoncture politique en plein dérapage. Le comportement obsessionnel du président Houphouët-Boigny est sans doute le facteur prépondérant : pour avoir les coudées franches, celui-ci invente des complots imaginaires, conçus comme un moyen efficace de neutraliser tout adversaire et rival éventuel. Les ambitions de toute une génération de cadres sont ainsi étouffées par une série d'arrestations : la domination houphouëtienne du pays est assurée. Le monde extérieur prend son pas sur celui de l'ancien pouvoir colonial, la France qui, préoccupée par les jeux stratégiques de la guerre froide, cautionne par son inertie les agissements du président ivoirien. Pour Kourouma, ce drame cauchemardesque est à la fois national et personnel. Non seulement le moment est difficile pour le pays, mais Kourouma est directement impliqué: il est incarcéré, avec un bon nombre de ses amis et connaissances. Il a expliqué, par la suite, à de nombreuses reprises, que cette expérience a déclenché chez lui le besoin d'écrire quelque chose sur la situation intolérable qu'il venait de vivre. Il parlera alors de ce besoin de témoigner pour ses compatriotes en termes de " nécessité ${ }^{2}$ ». Et certains des tapuscrits des Soleils porteront, en effet, sur la feuille de garde, de la main de Kourouma, la mention «Pour Lamine Diabaté et ses codétenus ${ }^{3}$ ", démontrant que son roman est bien le fruit de ce besoin de témoigner qu'il a identifié comme la première impulsion le poussant à écrire.

Le récit de l'émergence de ce premier roman s'apparente donc à un drame captivant rendu d'autant plus alléchant que le roman en question est marqué, dès avant les premières ébauches, du signe de l'impossibilité : impossible de concevoir son écriture étant donné la formation scientifique de Kourouma, étranger aux milieux littéraires et exerçant la profession d'actuaire; impossible de concevoir sa publication en Côte d'Ivoire étant donné la critique acerbe du président qu'il a l'intention d'exprimer ouvertement et, par conséquent, les risques de censure qui pèsent sur lui ; impossible, finalement, de concevoir un lectorat susceptible de lire un tel réquisitoire dans un pays dépourvu d'infrastructures institutionnelles (maisons d'édition, presse, et autres filières de communication) vraiment indépendantes de l'ancien pouvoir colonial, de ses valeurs et de ses intérêts.

Dans l'immédiat, Kourouma semble avoir fait fi de toutes ces bonnes raisons pour justifier l'abandon de son projet, préférant se tourner vers une question d'ordre pratique : quelle forme donner à ce désir de témoignage qui lui tenait tant à cœur ? Or, l'un des rares éléments du dossier génétique des Soleils est l'ensemble de documents (plan, notes et brouillon) consacré à « Houphouët-Boigny et le RDA ${ }^{4}$ ». L'analyse de ces documents a incité Jean-Francis Ekoungoun à conclure ceci: «Le futur auteur se 
propose [...] de rédiger, en première intention, non pas une fiction au sens littéraire du terme mais un essai sur l'histoire de la Côte d'Ivoire ${ }^{5}$. " Kourouma, pour sa part, a souvent expliqué qu'avant de fixer son objectif sur l'écriture d'une fiction, c'est la forme de l'essai qui l'avait d'abord attiré :

[...] j'ai voulu écrire quelque chose pour témoigner, pour dire que mes camarades étaient injustement arrêtés. Ceci dit, j'aurais pu écrire un essai mais un essai sur Houphouët à cette époque ne pouvait pas passer parce que Houphouët était très puissant et appuyé par la politique française et la politique de l'Occident. Donc un essai ne pouvait pas passer. Alors j'ai écrit un essai avec une fiction ${ }^{6}$.

Il est intéressant de noter que la solution trouvée par Kourouma est une solution hybride, "un essai avec une fiction", description qui se conforme beaucoup plus étroitement à la version tapuscrite du roman envoyée dans diverses maisons d'éditions, tant africaines que françaises, par les soins de Kourouma lorsqu'il cherchait un éditeur en 1966. Toujours est-il que cette description, " un essai avec une fiction", donne un aperçu de la double motivation qui l'animait au moment où il a pris la plume.

6 La suite de l'histoire éditoriale des Soleils se déroule à Montréal, sous l'œil vigilant de Georges-André Vachon, président du jury du Prix de la francité, prix que Kourouma avait emporté à condition d'accepter de retravailler son texte ${ }^{7}$. Cette histoire est bien celle d'un retranchement et cela dans les deux sens du terme. Kourouma supprimera une partie non négligeable de son texte (la partie qui était nourrie justement par les événements politico-historiques qui semblaient appeler l'écriture d'un essai); mais en ce faisant, il se retranche également derrière ses dernières défenses afin de mieux protéger ce qui lui tient le plus à cœur, la nature véritablement littéraire de son entreprise. L'importance accordée par Kourouma au projet littéraire, à la mise en forme d'une œuvre, à un travail d'ordre esthétique, n'a jamais été vraiment reconnue à sa juste valeur, comme si se consacrer à des préoccupations "littéraires » était une frivolité inavouable comparée à la tâche pressante de dénoncer des abus et fustiger un tyran, tâche qui, pour certains, serait même incompatible avec l'ambition littéraire. Il est vrai que cette demande de suppression des éléments "journalistiques», de ces passages qui permettaient aux lecteurs de reconnaître des personnages bien réels de la scène politique ivoirienne, peut être comprise comme une espèce d'ingérence de Vachon dans les affaires de Kourouma. Elle est doublement blessante pour certains commentateurs, puisqu'elle conforte la thèse de la domination postcoloniale: les exigences de Vachon se revêtiraient même des couleurs de la censure. Ainsi, le débat a tendance à basculer très rapidement vers une exploration approfondie de cet univers pragmatique de la politique (à la fois thème du roman mais également thème de la méta-histoire de son trajet vers la publication), à l'exclusion de la question beaucoup plus nuancée qui est celle du parcours littéraire suivi.

7 À ce jour, en effet, trop de critiques ont sous-estimé l'importance de l'aspect purement littéraire du travail effectué par Kourouma. Accorder trop d'importance au contexte politique du récit (ou de son histoire éditoriale) équivaut à en faire un simple " écrivant». Or, la célèbre distinction de Barthes entre un écrivant et un écrivain, formulée au moment même où Kourouma se débattait dans les marasmes de la vie politique en Côte d'Ivoire, s'applique directement à son cas : « L'écrivain accomplit une fonction, l'écrivant une activité [...] l'écrivain est un homme qui absorbe radicalement le pourquoi du monde dans un comment écrire ${ }^{8}$. " Et comme s'il cherchait à expliciter plus précisément le cas kouroumien, Barthes continue : 
[...] l'écrivain s'interdit existentiellement deux modes de parole [...]: d'abord la doctrine, puisqu'il convertit malgré lui, par son projet même, toute explication en spectacle [...]; ensuite le témoignage : puisqu'il s'est donné à la parole, l'écrivain ne peut avoir de conscience naïve : on ne peut travailler un cri, sans que le message porte finalement beaucoup plus sur le travail que sur le cri $[. . .]^{9}$. essai, tout comme il s'est vite rendu compte qu'entreprendre l'écriture d'une fiction exigerait un travail laborieux pour réprimer le cri intérieur, le façonner et le transformer en œuvre littéraire. Ainsi, ce choix liminal concernant la forme de son travail (essai ou fiction?) doit être compris comme le premier pas dans un processus dans et par lequel des préoccupations littéraires s'imposent peu à peu, au fur et à mesure que l'inspiration politico-historique s'estompe. Il aurait, sans doute, préféré éviter la dernière campagne rédactionnelle montréalaise et les changements de dernière heure demandés par Vachon, mais en acceptant de s'y plier, il ne fait que continuer le travail de façonnage qui caractérise le véritable écrivain.

Il serait malencontreux de voir dans cet argument une tentative d'amoindrir l'élan initial du projet kouroumien. Le cri qu'il voulait pousser venait du cœur. Mais ce qui nous enseigne le plus sur l'attitude de Kourouma, c'est la manière dont il refuse de se laisser dominer par les événements, pour aussi cruels qu'ils soient. Au lieu de pousser des cris de démagogue, il cherche à comprendre; il se met à verbaliser le monde qui l'entoure et à se l'expliquer. Le texte sur « Houphouët-Boigny et le RDA » est loin d'être une diatribe contre les méfaits du régime houphouëtien. Calme et posé, il aurait sans doute fait moins de remous s'il avait été publié que le récit des tortures infligées par Houphouët-Boigny et sa sœur qui figurent dans l'avant-texte du roman. Cette constatation nous permet de mieux apprécier l'ambivalence inhérente à son projet d'écriture. Certes, Kourouma voulait témoigner en 1963 contre les abus de pouvoir d'un régime liberticide, mais ce qu'il voulait dénoncer, sa véritable cible, se situait ailleurs, historiquement et géographiquement. Kourouma était assez lucide pour comprendre que le comportement d'Houphouët-Boigny n'était qu'un cas de figure parmi d'autres et que la grande tragédie que vivait son pays était celle, infiniment renouvelable, de l'exploitation de l'homme démuni par l'homme en situation de tout se permettre. Cela explique pourquoi Kourouma semble avoir eu tant de mal à en vouloir personnellement (ou à la personne même) du président de la République. Vu sous cet angle, HouphouëtBoigny n'était qu'un pantin mû par des forces qu'il était incapable de maîtriser. Dans un texte peu connu écrit en 1998, Je témoigne pour l'Afrique, Kourouma revient sur ce thème que l'on pourrait identifier comme l'injustice majeure qu'il s'est consacré à dénoncer par son travail littéraire: «À l'Afrique sans défense, dans l'Afrique loin de tous les yeux discrets j'ai vu ce qu'un homme fait d'un homme sans défense et loin de tous les yeux discrets $^{10}$." Dès qu'on relativise l'actualité politique qui fournit le contexte d'émergence des Soleils, la notion de "la nécessité d'écrire » qui animait Kourouma à l'époque prend une autre allure. De facteur décisif dans l'écriture de son premier roman, «la nécessité » devient le moteur de la production littéraire de toute une vie, ce que Kourouma semble confirmer dans une interview accordée peu de temps avant son décès :

J'ai dit que Les Soleils des indépendances a été écrit par nécessité. Les autres aussi, c'est par nécessité, parce que je m'étais aperçu que les Occidentaux parlaient de tout ce que les communistes avaient fait comme crimes en Orient. Et en France, on parle des crimes que les Allemands ont faits pendant quatre ans d'occupation. Tous les ans les Français en parlent et même cette année encore vous verrez dans des 
livres qui sortent, on parlera de l'Occupation. Alors ça m'a révolté. J'ai dit : « Mais quand même, nous qui avons vécu cinquante-cinq ans de colonisation, qu'est-ce que nous avons à dire?» Et j'ai voulu écrire moi-même cette tragédie. [...] Monnè, outrages et défis c'est un peu ça, tu vois. Tandis qu'En attendant le vote des bêtes sauvages c'était aussi parce que la guerre froide a fait beaucoup, beaucoup, beaucoup de mal en Afrique. [...] La guerre froide a provoqué de graves désordres chez nous, justement parce que cela a permis aux dictateurs de se maintenir ${ }^{11}$.

10 Les méfaits d'Houphouët-Boigny, les crimes de la colonisation, les conséquences néfastes de la guerre froide en Afrique, le sort des enfants soldats sont autant d'avatars de ce thème primordial qui est celui de l'exploitation de l'homme par l'homme. Le cri que Kourouma avait envie de pousser en 1963 se traduit ainsi en une position existentielle: il ne pourra rester silencieux face à de telles injustices; l'écriture, façonnage du cri, sera désormais son exutoire.

11 Le simple fait d'identifier une source d'inspiration pérenne n'équivaut pourtant pas à une analyse de la genèse auctoriale de l'auteur en question. Pour respecter la démarche caractéristique de la génétique textuelle, toute tentative d'élaborer le «devenir auctorial » doit donner la priorité à un examen des traces matérielles résultant du travail accompli pendant la production des textes qui constituent une œuvre. Ces traces matérielles peuvent être de plusieurs types : des bribes et des résidus de campagnes rédactionnelles évidemment, mais aussi des traces d'une réflexion susceptible de nourrir le travail en cours - lectures annotées ou commentées, soit sur le sujet à traiter dans un travail éventuel, soit sur la manière d'aborder la mise en forme, ces aspects techniques et stylistiques de la praxis de la narration. Elles peuvent également prendre la forme de commentaires après coup sur un travail déjà accompli : des interventions dans des débats ou colloques, des interviews, des textes de tout genre explicitant le travail proprement littéraire et destinés à la presse écrite, à des revues universitaires et autres. Bref, tout cet arrière-pays dont l'arpentage permet de mieux suivre l'acheminement d'une pratique scripturale et la réflexion menée sur cette pratique.

De cela découle une observation importante. Lorsqu'on cherche à comprendre la « genèse » d'un écrivain, le travail qu'il/elle accomplit pour assurer l'émergence d'une première publication ne saurait avoir de statut particulier. Certes, sur le plan biographique, il se peut qu'une première publication ait une grande signification dans la vie d'un auteur, accordant reconnaissance et parfois une sorte de consécration, facilitant aussi la possibilité d'une certaine continuité dans la carrière littéraire envisagée. Mais, sur le plan génétique, une première publication peut difficilement prétendre à un statut déterminant. Ce qui importe, c'est le modus scribendi de l'écrivain, sa façon de travailler, les efforts fournis pour se plier aux exigences de la mise en forme telle qu'il/elle la conçoit. Le dossier génétique du " devenir écrivain » de Kourouma est, à cet égard, assez fourni.

14 Étant donné ses objectifs non conformistes, sa revendication de la culture malinké, son statut de « sujet postcolonial » et sa situation marginale par rapport à ce vaste champ de pratique culturelle qu'est la littérature française, il n'est pas étonnant que son écriture ait eu un potentiel énorme pour bouleverser le statu quo. En l'occurrence, il s'agissait de l'invention d'une nouvelle forme d'expression. Comme l'a expliqué Daniel Delas: «Quand le réel devient innommable, l'écrivain doit affronter le choix d'un nouveau discours, d'une nouvelle écriture ${ }^{12}$." Cela est doublement le cas pour Kourouma, qui se voulait le témoin d'une réalité innommable, tout en étant conscient que le français était un outil inadéquat à la tâche qu'il s'était donnée. Voulant parler de 
phénomènes bien concrets, Kourouma s'est retrouvé d'emblée confronté à toute une série de défis formels qu'il ne pouvait résoudre qu'en trouvant sa "posture d'écriture ${ }^{13}$ ", compatible avec une conception toute personnelle de la création littéraire. Sans forcément vouloir «devenir écrivain » ou «faire de la littérature », il s'est propulsé au cœur même d'une recherche on ne peut plus littéraire. Il ne théorisait pas ces questions, mais voulait tout simplement rester fidèle à sa vision du monde, à ses assises culturelles malinké et néanmoins s'arroger le droit de s'aventurer dans le domaine de chasse gardée de la littérature française. La solution qu'il trouve, à la fois posture d'écriture et stratégie de mise en forme esthétique, sera d'opérer une hybridisation des langues française et malinké, la soi-disant malinkisation de la langue française qui a fait couler tant d'encre.

15 Cette stratégie n'est pas fortuite. Elle est le fruit d'une réflexion mûrie, menée pendant de longues années et continuée bien après la publication des Soleils, sur les difficultés d'un Africain désireux d'exprimer les réalités de sa propre culture dans une langue qui n'est pas la sienne. Déjà, en 1970, année de la publication des Soleils en France, Kourouma a saisi l'occasion d'une de ses premières interviews pour s'exprimer sur le dilemme de beaucoup d'écrivains obligés de négocier leur « francophonie »:

La langue française est entourée d'une grande dévotion, objet d'une sorte de fétichisme stérile qui a hypothéqué jusqu'à ces dernières années les travaux d'écrivains non français, mais possédant en elle leur unique moyen d'expression. On a voulu conserver la langue dans ses fortifications grammaticales et ses clichés. Bien parler français, un point c'est tout... Au fond, ce n'est pas l'alphabétisation qui est un problème. Alphabétiser les masses est relativement facile. C'est leur donner le moyen d'accorder la sensibilité à un outil d'expression. Les Anglais n'ont pas couvé leur langue comme les Français et c'est une bonne chose. Et croyez-moi, ce « néo-créole » au Nigéria a donné naissance à une expression écrite qui a toutes les caractéristiques d'une vraie littérature. Laisser s'enrichir une langue au contact d'un peuple dont elle n'est pas issue, ne débouche pas forcément sur un dépérissement de cette langue ${ }^{14}$.

Les difficultés rencontrées par Kourouma dans les années soixante, lorsqu'il essayait de trouver un éditeur pour son roman, ont été exacerbées par un scepticisme très prononcé et assez généralisé quant à sa compétence en tant que locuteur/scripteur de français. Les lecteurs professionnels, qui avaient pour tâche d'évaluer le roman, ne comprenaient pas ses audaces stylistiques, les considérant comme des fautes grammaticales ou des fautes de goût. Le biographe de Kourouma, Jean-Michel Djian, rend compte de la réaction de quelques-uns des premiers lecteurs: "Il [Kourouma] n'essuie que des refus, en particulier ceux du Seuil et de Présence africaine, qui trouve son texte "mal écrit". François-Régis Bastide, lecteur influent de la maison de la rue Jacob, l'a refermé au bout de cinq feuillets ${ }^{15}$. » Kourouma a évoqué cette expérience quelques années plus tard, dans une interview pour Jeune Afrique Économie :

La première chose qu'on fait c'est de prendre contact avec des éditeurs africains. Ils m'ont répondu que ce n'était pas rédigé en bon français, qu'il fallait d'abord que j'apprenne à écrire dans cette langue [rires]. [Présence africaine commençait à être subventionné par les États africains...] Mon livre, dans une partie expurgée, comportait beaucoup d'allusions à peine voilées, à la politique en Côte d'Ivoire, au régime, aux tortures. Alors évidemment Présence africaine m'a dit, «Avant de critiquer les États africains, avant d'attaquer le président, apprenez donc à écrire en français » [rires $]^{16}$. 
17 Kourouma reviendra inlassablement sur ce thème de l'inadaptation de la langue française dans des conférences, colloques et autres événements culturels, dont voici un échantillon :

- «Les littératures négro-africaines », Lomé, novembre 1991;

- colloque à Rome, décembre 1992 ;

- «Poètes et penseurs comme précurseurs et porteurs d'espoir au cœur du changement en Afrique », Tübingen, novembre 1993 ;

- « Pour une francophonie des peuples, des cultures et des dieux », Abidjan, mars 1994 ;

- «Le processus d'africanisation des langues européennes », Montpellier, décembre 1994 ;

- «Les dits et les non-dits », Wellesley College, Massachusetts, 1999,

- discours prononcé lors de la conférence du Centre international des études françaises, Abidjan, décembre 2002.

Le problème évoqué dans ces diverses interventions est principalement linguistique, mais le questionnement porte aussi sur des questions d'ordre social et culturel. Kourouma s'indigne contre une presse écrite (en français) qui se trouve incapable de rendre compte d'événements quotidiens se produisant dans les communautés ivoiriennes, faute de pouvoir traduire en français des expressions courantes dans la culture indigène. Jean-Michel Djian cite l'exemple de l'intervention de Kourouma à la conférence d'Abidjan en mars 1994 où, à propos de sorcellerie, ce dernier déplore le fait qu'un tribunal puisse condamner un individu pour un crime qui n'est pas «nommable » en français : «Si le français est la langue officielle de la Côte d'Ivoire, il est inadmissible que les faits pour lesquels des citoyens sont jugés ne soient pas nommables dans leur langue nationale ${ }^{17}$. » Il explique la difficulté dans les termes suivants :

Le négro-africain, partout et dans tout ce qu'il fait, n'oublie pas qu'il appartient à une communauté que chacun de ses actes compromet. Tout possède une force immanente qui peut venger les victimes des injustices et des mensonges, les tuer, les torturer; toutes les choses qui ont été détruites sans nécessité, sans raison par un membre de notre communauté peuvent réagir sur nous. C'est pourquoi le négroafricain évolue dans un monde bâti de signes à interpréter, de forces à maîtriser pour évoluer. Il est lié avec les membres de la communauté, il ne peut abandonner et espérer évoluer, réussir comme individu ${ }^{18}$.

Une réflexion soutenue sur ce sujet accompagnera le travail littéraire de Kourouma tout au long de sa vie. Il citera l'anthropologue Louis-Vincent Thomas dans un discours inédit prononcé à Abidjan en 2002, où apparaît sa connaissance profonde des particularités lexicales et syntactiques des langues africaines. En 1990, il accepte même d'écrire une préface pour un projet de livre de Mariette Meunier-Crespo, Le Français populaire d'Abidjan, tiré d'une thèse de doctorat en linguistique. Le livre ne paraîtra pas, toutefois un premier jet de la préface envisagée existe dans les archives. Kourouma profite de l'occasion pour réitérer sa frustration :

Le français généré pour une civilisation de substrat chrétien et latin et forgé et poli par une littérature écrite exprime mal certains sentiments et aspects des richesses culturelles de ces peuples dont la littérature est orale et la religion de base animiste. Il en résulte, même pour les Africains qui ont la parfaite maîtrise du français, une certaine frustration... Ils ne se sentent pas à l'aise dans une langue censée être leur langue nationale ${ }^{19}$.

20 À Montpellier, en 1994, Kourouma ira jusqu'à suggérer tout un programme pour favoriser l'insertion de mots africains dans les dictionnaires de langues européennes, mû par une volonté de défense et illustration des langues africaines. 
21 Cette préoccupation quasi obsédante, issue d'une réflexion permanente sur des questions concernant l'expression culturelle, fait écho aux questions que Kourouma a dû se poser lorsqu'il a entrepris d'écrire une œuvre de fiction en 1963. Transposée dans le domaine littéraire, l'inquiétude de Kourouma se transforme en une interrogation sur le style à adopter. Il explique que sa méthode de travail et sa décision d'exprimer l'univers de Fama de l'intérieur de son personnage, en employant sa propre voix et ses propres habitudes langagières, ne pouvaient pas être une simple question de traduction, mais impliquaient la création d'une nouvelle langue :

Ce que j'ai compris intuitivement, c'est que si je faisais parler mon héros en français classique, il ne m'apparaissait pas correspondre à ce que je voulais : la façon dont il pensait, élaborait et classait ses concepts, tout cela ne venait pas [...]. Il ne s'agit pas de traduire mais bien de saisir un sens, un rythme, une façon de percevoir et d'exprimer, et de rendre tout cela en français [...]. Mon style n'est pas une traduction mot à mot. Ne faire que traduire serait facile; or ce que je fais réclame beaucoup de temps et de peine; il m'est arrivé de réfléchir une journée entière à une phrase jusqu'à ce qu'elle coule jusqu'à ce qu'elle trouve la forme exacte qui convienne. Il ne s'agit pas de traduire mais bien de saisir un sens, un rythme une façon de percevoir et d'exprimer ${ }^{20}$.

Il reparlera de ce problème de création dix ans après cette interview, lorsqu'il interviendra dans un colloque organisé à Wellesley College, dans le Massachusetts. Là encore, il fait allusion aux problèmes linguistiques qui ont été à l'origine de ses choix littéraires et stylistiques :

Mon projet n'était pas de malinkiser le français mais de faire de sorte qu'un lecteur vive le cheminement de la pensée du personnage, qu'il voit ce que voit le personnage et de la façon qu'il le voit, qu'il sente ce qu'il sent et de la façon qu'il le sent.

Comment aboutir à un tel résultat? Qu'ai-je entrepris pour le réussir?

D'abord en agissant sur la structure du langage. Dans chaque langue les mots ont en général une succession propre. J'ai appliqué dans le français la succession des mots en malinké. Dans le malinké comme dans toutes les langues orales l'on fait très peu usage des pronoms: les substantifs, les syntagmes sont très souvent répétés. J'applique la même méthode en français. Ce qui fait que le texte comporte des phrases courtes. Mais c'est dans la recherche des mots qu'a lieu la plus grande innovation. Il y a beaucoup de réalités africaines [qui] n'ont pas de nom en français. Il faut trouver le mot juste qui les exprime. Rarement on trouve ce mot juste. On procède par approximation, en usant de plusieurs mots qui ont des sens proches, des synonymes. Une accumulation des synonymes pour exprimer une réalité signifie qu'aucun des mots, aucun pris seul ne l'exprime en totalité, ne l'épuise entièrement. Il y a beaucoup d'expressions malinké qui sont intraduisibles en français. Et cela pour une raison simple. Les malinkés sont des négro-africains animistes. Chaque objet a une âme. Donc tout objet peut se mouvoir comme un animal, parler comme un homme. Des expressions issues d'une telle conception des objets sont intraduisibles en français. Il faut passer par mille détours pour asseoir cette réalité. Et quand on le réussit on jubile. C'est cet ensemble de recettes qu'on a appelé malinkiser le français ${ }^{21}$.

La posture d'écriture trouvée par Kourouma a choqué certains lecteurs et scandalisé d'autres, comme si son objectif avait été de porter atteinte à la langue française. Elle répondait, au contraire, à une autre forme de nécessité, toute scripturale celle-là, qui n'était rien d'autre que la recherche, pour lui malinké, du mot juste. Cette activité, cette rumination constante sur le "comment dire" sont, au fond, une forme de recherche dans et par laquelle l'écrivain se construit. 
'est pas étonnant donc que la fascination de Kourouma pour tout ce qui touchait à l'expression culturelle, et ses efforts pour créer une interlangue capable de favoriser une meilleure communication interculturelle, aient eu pour corollaire une passion pour les dictionnaires. Dans une interview enregistrée peu de temps avant son décès, Kourouma n'a pas hésité à identifier quels étaient les livres de sa bibliothèque auxquels il tenait particulièrement :

Je vous réponds sans la moindre hésitation : à mes dictionnaires. Je possède sur mon ordinateur tous les dictionnaires français imaginables. Du dictionnaire de l'Académie française au Littré, en passant par les dictionnaires des Curiosités, le Dictionnaire universel des synonymes de Guizot, et les autres usuels. Il a fallu équiper le disque dur de mon ordinateur de capacités énormes afin de pouvoir conserver tous ces dictionnaires ${ }^{22}$.

En dehors des exemplaires stockés sur le disque dur de son ordinateur, Kourouma possédait également une collection impressionnante de dictionnaires et de livres de référence, des manuels de stylistique et des livres sur divers aspects de la linguistique appliquée. La bibliothèque de Kourouma a été transférée dans les archives de l'Institut Mémoires de l'édition contemporaine et un inventaire de cette collection a pu être établi ${ }^{23}$. Étant donné que la bibliothèque de Kourouma a été dilapidée au moins deux fois au cours de sa vie (une fois par effraction et vol, et une deuxième fois lors d'un déménagement), on ne peut savoir si cette collection est exhaustive. Toujours est-il qu'elle contient quelque 86 volumes et constitue un parfait outil de travail pour l'écrivain autodidacte. Un bon nombre des volumes portent les traces d'une lecture attentive: des traits verticaux dans les marges et des soulignements indiquent des éléments qui ont retenu l'attention de Kourouma lors de sa lecture. Dans le livre de Marcel Cressot, Le Style et ses techniques, par exemple, Kourouma marque d'un trait le passage suivant, qui se conforme très précisément à l'importance qu'il accorde luimême au rythme de la phrase :

Il est bien évident que l'écrivain, ou tout simplement l'usager, quand il écrit une phrase ne se livre pas à ces calculs. Une pensée arrivée à sa maturité se construit elle-même le rythme dont elle a besoin pour son expression totale. L'instinct, l'esprit critique avertissent le véritable écrivain de l'opportunité de ce rythme spontané. Que de corrections chez les bons auteurs reposent uniquement sur des intentions rythmiques ${ }^{24}$.

Et lorsque Pierre Guiraud remarque dans son livre, La Stylistique :

M. Barthes montre excellemment comment l'emploi du passé défini ou du «il» romanesque n'a pas d'autre fonction que d'assurer «ce système de sécurité des Belles Lettres » hérité de Flaubert à travers Maupassant, Zola, Daudet, se perpétue assez paradoxalement - dans la littérature prolétarienne et révolutionnaire où il continue à assumer cette valeur de signal.

Kourouma ajoute laconiquement dans une note marginale : « ou parce que les écrivains sont souvent des autodidactes ${ }^{25}$ ». L'autodidacte qu'était Kourouma a lui-même ressenti puis rejeté cette tentation de l'écriture "classique ». Son instinct l'a conduit à opter pour une approche stylistique plus audacieuse, ce qui ne l'a pas empêché pour autant d'étudier assez systématiquement ses outils de travail. Les autres livres qui portent les traces d'une lecture attentive sont :

-Walter von Wartburg et Paul Zumthor, Précis de syntaxe du français contemporain, Berne, Éditions Francke, 1973.

- Frédéric Deloffre, La Phrase française, Paris, SEDES, 1979.

- P. Barucco, Éléments de stylistique, Paris, Éditions Roudil, 1972.

Continents manuscrits, $10 \mid 2018$ 
- Henri Sensine, L'Emploi des temps en français, Paris, Éditions Payot, 1966 (Kourouma possédait une deuxième édition de ce texte, datant de 1981).

- Jean-Pierre Colignon, La Ponctuation : art et finesse, Paris, Éditions Eole, 1981.

- Mariette Meunier-Crespo, Des données empiriques à l'objet d'étude : la constitution d'un corpus de démodialectologie (français populaire d'Abidjan), université Jean-Moulin, Lyon III, thèse en 2 volumes.

L'existence de cette collection de dictionnaires, et l'emploi que Kourouma semble en avoir fait, démontrent une véritable volonté d'apprentissage et de formation dans les compétences nécessaires pour celui qui chercherait à bien écrire. Sans formation littéraire formelle, Kourouma utilise les moyens du bord pour se renseigner sur le métier d'écrivain et mieux comprendre les normes et les écarts linguistiques et stylistiques du français écrit. La malinkisation de la langue française qui en résultera dans le cas des Soleils, tout comme les prouesses stylistiques dont il fera preuve dans ses autres romans, ne peuvent donc aucunement être associées à des défauts de compétence en français, reproche que beaucoup de ses premiers lecteurs se sont permis de lui faire.

Le devenir auctorial de Kourouma est à saisir dans ce processus de réflexion sur des problèmes d'expression, cette lente maturation d'une pensée qui s'interroge sur la question "comment écrire»? Les documents d'archives, les manuscrits et tapuscrits ainsi que la correspondance de Kourouma confirment que, pour lui, "devenir écrivain " ne pouvait avoir de sens que dans une pratique de l'écriture menée jour après jour sur toute une vie. La correspondance de Kourouma nous permet de glaner quelques renseignements sur sa façon de travailler. Il est assidu et régulier, malgré ses occupations professionnelles qui ne lui laissent que peu de temps libre. Dans une lettre à un enseignant de la faculté des Lettres de Nice en 1978, il écrit : «Depuis cinq ans je travaille sur un roman qui sera en trois volumes. Mais c'est long et difficile; j'écris au maximum deux heures par jour, pas plus ${ }^{26}$.» Pour alléger un travail sans doute assez solitaire, mais aussi par gentillesse naturelle, il s'ouvre facilement à toute personne qui s'intéresse à son travail, envoyant des textes à des lecteurs et appréciant l'opinion d'amis de longue date, telle Arlette Chemain, à qui il écrit vers le début de 1977 : «Je voudrais vous envoyer, dès que je l'aurai achevé et dactylographié (vraisemblablement à la rentrée prochaine) le manuscrit du premier volume du roman sur lequel je travaille depuis quelques années. Il sera en trois volumes. Je souhaiterais connaître quelques critiques sur ce livre avant de le proposer à mon éditeur ${ }^{27}$. " Il lui arrive également de partager avec ses correspondants le détail de ses méthodes de travail :

Je m'efforce en écrivant, autant que je peux, de présenter les faits dans la forme, l'ordre le sentiment dans lequel le narrateur le perçoit ou l'exprime, de conserver toujours à l'événement la structure qu'il a pour le narrateur; je ne fais pas dire le narrateur : il pense, sent représente.

30 Il continue en faisant référence à son habitude d'effectuer des changements de perspective/ de voix à l'intérieur du récit: «l'emploi d'aucuns de ces procédés n'est d'ailleurs systématique, ce qui l'est c'est le jugement de mes oreilles. Je lis à haute voix ce que j'ai écrit et l'arrange jusqu'à ce que satisfaction soit donnée à mon ouï ${ }^{28}$ ".

31 Naturellement, il se peut que le dossier génétique de telle ou telle œuvre fournisse une deuxième source de commentaires sur l'écriture en gestation, s'il contient des éléments portant sur cette méta-activité qu'est la réflexion consacrée au processus scriptural en cours de route. Assez paradoxalement, le dossier génétique des Soleils ne contient que 
très peu de traces de ce genre de réflexion. Bien que nous sachions que Kourouma travaillait et retravaillait ses textes sans cesse, le dossier génétique des Soleils ne livre que peu de traces d'un tel labeur. De ce point de vue, en effet, le manuscrit du roman peut surprendre par le degré de finition qu'il exhibe. Il comporte, certes, des biffures, des rajouts et de nombreux exemples de réécriture, mais, malgré ces modifications, l'écrivain propose un texte " complet ", parfaitement lisible. Kourouma a déjà trouvé le personnage de Fama et la voix qui lui permettra de développer son récit avec confiance. Par contraste, le dossier génétique de son deuxième roman, Monnè, outrages et défis, est riche en avant-textes et en documents issus de diverses campagnes rédactionnelles menées sur un laps de temps très long. Il est composé d'une quinzaine de cahiers manuscrits et d'un bon nombre de tapuscrits (qui sont souvent des exemplaires multiples du même texte). Naturellement, c'est dans les cahiers manuscrits que le véritable travail d'écriture se déploie, le tapuscrit ne voyant le jour qu'au moment où le texte est considéré comme suffisamment " terminé » pour mériter un autre support de lecture. Or, les cahiers abondent en faux départs, recommencements et reprises, hésitations en tout genre, qui confirment que Kourouma se cherchait, autant sans doute dans la recherche du thème qu'il voulait aborder que dans l'écriture même.

Deux cahiers en particulier comportent des commentaires susceptibles de nous informer sur sa pratique scripturale: le cahier Manolux beige et le cahier Manolux vert $^{29}$. Pendant les années soixante-dix, Kourouma avait pris l'habitude d'écrire sur les feuillets recto des cahiers qu'il favorisait à l'époque comme support, laissant vierge le feuillet verso. Cela lui permettait d'utiliser au besoin ces feuillets verso pour ajouter des remarques sur le travail en cours : souvent elles consistaient en de simples résumés une phrase - du thème abordé dans la narration se déroulant sur le feuillet en face; il pouvait également s'agir de commentaires sur le plan à suivre, la structuration du récit en chemin ou à venir; il ajoutait fréquemment des rappels et des conseils adressés à lui-même, des réflexions plus ou moins développées sur la nature même de l'écriture et les effets qu'il cherchait à obtenir. Si besoin était, Kourouma pouvait aussi se servir de ces feuillets vierges pour réécrire une phrase ou un passage de son récit. Voici une sélection non exhaustive des remarques, commentaires et auto-conseils sur le travail en cours, tirés de ces deux cahiers :

\section{Cahier Manolux beige}

fo $7 \mathrm{v}$ :

Méthode : N'écrire le deuxième jet

doit être d'abord travailler [sic] sur des brouillons

volants avant être copié. Cela est indispen

sable il permettra l'exploitation de plusieurs

pages à la fois du premier jet

fo $8 \mathrm{v}$ :

- le livre doit être celui de l'échec

- chaque paragraphe doit se terminer par

un espoir rapide dont [sic] le prochain

paragraphe commencera d'effacer avant

de commencer

fo $9 \mathrm{v}$ :

les mauvaises langues

fo $11 \mathrm{v}$ :

Les méchancetés de Djigui

fo $14 \mathrm{v}$ :

Méfaits toujours (le chemin de fer) 
fo $16 \mathrm{v}$ :

Djigui et le marabout Yacouba

fo $22 \mathrm{v}$ :

La misère du peuple

fo $27 \mathrm{v}$ :

Djigui lui disait que la puissance peut

tout contenir sauf la vérité, la vérité sans

dissimulation, elle peut tout sauf faire ce que

le puissant veut. Si tu oublies ces choses

tu ne seras jamais

Comme caractère de Tieba en faire un fataliste.

E'est un peu moi. Tieba c'est moi.

fo $49 \mathrm{v}$ :

Faire l'écriture Écrire en pensant en malinké

surtout.

fo $103 \mathrm{v}$ :

Dans un roman les faits ne doivent pas être reportés. Sinon ça devient reportage ; mais vécu dans une conscience. C'est pourquoi

fo $104 \mathrm{v}$ :

Pourquoi ne pas montrer la réussite de Bema par une nouvelle élection qui se terminera par une victoire des opposants du RDA.

fo $108 \mathrm{v}$ :

Dans un roman les choses ne doivent pas être servies crues, brutes - Il faut un peu d'éloignement, ou que les choses arrivent comme un écho après une réflexion dans une conscience.

fo $109 \mathrm{v}$ :

Il faut là présenter les sacrifices et les consultations des sorciers et des marabouts.

fo $115 \mathrm{v}$ :

Peut-être faire expliquer la nouvelle politique, la nouvelle situation par Bintou.

\section{Cahier Manolux vert}

fo $2 r$ :

le roman ce n'est pas le fait. Il est menteur logique, trompeur irréaliste. C'est le vécu comment il est reçu qui est vrai, réaliste, saisissable - les faits sociaux, le droit, le domaine du roman c'est donc ce vécu dans cette conscience - les faits ne sont pas importants et secondaire [sic] que par l'importance la signification que la conscience leur donne.

Donc dans le roman ne relevez les faits que dans la mesure ils [sic] sont importants pour le narrateur

fo $79 \mathrm{v}$ :

plus une chose nous porte du déplaisir mieux elle mérite d'être retenue

fo $80 \mathrm{v}$ :

Rêver de la situation de Nixon. Se rappeler toujours que certaines choses ne doivent être présentées que renversées, éloignées

$f^{\circ} 82 \mathrm{v}$ :

les étoiles et les nuages qui venaient et partaient quand la lune apparaissait tout cela occupait son cœur et son esprit et cela valait beaucoup mieux que l'oppression, et une chose enveloppait ces choses et ne le quittait jamais, c'était le sentiment de jouer un grand rôle politique.

Mouraient pour rien, disons sans peur pour le vent, le vent du rien. Et c'était d'ailleurs toujours le vent

fo $85 \mathrm{v}$ :

Ne pas perdre de temps sur la forme et la mise en scène

fo $89 \mathrm{v}$ :

Rendre l'opération beaucoup plus tragique 
fo $92 \mathrm{v}$ :

les choses doivent être vécues dans une conscience

Ici Bintou

À la lumière de sa passion pour la communication interculturelle, perceptible dans ses nombreuses interventions de par le monde, les manuscrits de travail et la correspondance démontrent à quel point le parcours de Kourouma a été résolument celui d'un écrivain en train de se construire. Il est possible, évidemment, de présenter Georges-André Vachon comme son sauveur, celui qui aurait su distinguer son talent lorsque l'establishment littéraire ne voyait en lui qu'un malotru peinant avec la concordance des temps. Dans une certaine mesure, ce schéma hâtif a été quelque peu conforté par Kourouma lui-même, lorsqu'il a exprimé sa gratitude envers l'universitaire québécois, alors décédé, dans un hommage publié en 1995, «Vachon, l'ami qui m'a fait ${ }^{30}$ ». Kourouma a beau écrire, avec sa générosité habituelle, "C'est l'ami à qui je dois ma carrière d'écrivain [...] c'est l'ami qui m'a fait », il n'en reste pas moins vrai que la genèse d'un auteur ne peut pas se limiter à l'histoire de ses démêlés avec des maisons d'édition et le trajet qu'il a suivi vers une première publication ${ }^{31}$. La véritable genèse de Kourouma écrivain s'inscrit dans une pratique de l'écriture et se déploie sous une double contrainte: linguistique, d'abord, puisque sa naissance à l'écriture s'apparente à une lutte intense pour faire émerger une langue idiolectale nourrie de sa double appartenance aux cultures malinké et français; politico-culturelle ensuite, parce que son statut d'écrivain francophone postcolonial représente un deuxième obstacle à ses tentatives de faire entendre sa voix. Il a fallu l'effort de toute une vie pour surmonter ces difficultés. Le «devenir écrivain » de Kourouma n'est au fond donc rien d'autre qu'un synonyme de la façon toute personnelle qu'il a choisie pour s'engager dans cette lutte.

\section{ANNEXES}

Inventaire des 'Dictionnaires' de Kourouma

$\underline{\text { KOU BP } 1 / 4}$

Pierre Gilbert, Dictionnaire des Mots Nouveaux, Paris: Hachette/Tchou, 1972

Jean-Paul Colin, Dictionnaire des Difficultés du français, Paris: Hachette/Tchou, 1970

Daniel Delas et Danièle Delas-Demon, Nouveau Dictionnaire Analogique, Paris: Hachette/ Tchou, 1971

Hector Dupuis et Romain Légaré, Dictionnaire des synonymes et des antonymes, Montréal/ Paris: FIDES, 1975

Petit Larousse 1965

Jean Girodet, Dictionnaire du bon français, Paris: Bordas, 1981

Georges Niobey (dir.), Nouveau dictionnaire analogique, Paris: Larousse, 1980 
Adolphe V. Thomas, Dictionnaire des difficultés de la langue française, Paris: Larousse, 1971

Christian Baylou et Paul Fabre, Grammaire systématique de la langue française, Paris:

Nathan, 1978

Georges Jean, Le Plaisir des mots, Paris: Gallimard, 1982

Jacques Drillon, Traité de la ponctuation française, Paris: Gallimard, 1991

André Sève, Ortho: Dictionnaire Orthographique et Grammatical, Chambéry: EDSCO, 1950

Maurice Maloux, Dictionnaire des Proverbes, Sentences et Maximes, Paris: Larousse, 1960

$\underline{\text { KOU BP 2/4 }}$

Dictionnaire sans titre relié de façon artisanale

M.M. Dubois, Dictionnaire Moderne Français-Anglais; Anglais-Français, Paris: Larousse (collection Saturne), 1960

Equipe IFA, Inventaire des particularités lexicales du français en Afrique noire, Québec: AUPELF, 1983

Paul Rouaix, Trouver le mot juste: Dictionnaire des idées suggérées par les mots, Paris: Armand Colin, 1897

Georges Niobey (dir.), Nouveau dictionnaire analogique, Paris: Larousse, 1986

Emile Genouvrier, Nouveau dictionnaire des synonymes, Paris: Larousse, 1992

Emile Genouvrier, Nouveau dictionnaire des synonymes, Paris: Larousse, 1977

François Suzzarani, Dictionnaire Marabout analogique, Alleur: 1984

Albert Dauzat, Nouveau dictionnaire étymologique et historique, Paris: Larousse, 1971

Emile Genouvrier, Nouveau dictionnaire des synonymes, Paris: Larousse, 1977

Joseph Hanse, Nouveau dictionnaire des difficultés du français modern, Paris: Duculot, 1983

René Thimonnier, Code orthographique et grammatical, Paris: Marabout Hatier, 1970

Jean Dubois (dir.), Lexis: Dictionnaire de la langue française, Paris: Larousse, 1975

Joseph Hanse, Nouveau dictionnaire des difficultés du français moderne, Paris: Duculot, 1983

Walter von Wartburg et Paul Zumthor, Précis de syntaxe du français contemporain, Bern Editions Francke Berne, 1973

Sylvie Weil et Louise Rameau, Trésor des expressions françaises, Belin 1981

Geo Sandry et Marcel Carrère, Dictionnaire de l'argot moderne, Paris: Mireille Ceni, 1973

KOU BP 3

Roger Pillaudin (dir.) Ecrire... Pour quoi? Pour qui?: Presses Universitaires de Grenoble, 1974

Nicole Everaert-Desmedt, Sémiotique du récit, Bruxelles:Editions universitaires, 1988

Inventaire des particularités lexicales du français d'Afrique noire, [dédicacé 21/1/88; signature illisible]

Louis Chaufferin et Fernand Hebert, L'Anglais commercial, Paris: Larousse, 1928 
André Goosse, La Nouvelle Orthographe, Gembleux: Duculot, 1991

Pascal Krop, Tu fais l'avion par terre: Dictionnaire franco-africain, J-C.Lattès, 1995

E.Legrand, Stylistique française, Paris: Ed. J.de Gigord, 1986

H.Manseau et F.Malka, Hugo Plus: Guide d'utilisation, 1993

U.Lacroix, Dictionnaire des Mots et des Idées, Paris: Nathan, 1961

Jean-Pierre Colignon, La Ponctuation: art et finesse, Paris: Editions Eole, 1981

Paul Robert (dir.) Micro-Robert: Dictionnaire du français primordial,Paris: Robert,1983

(Tome 1: A à L: Tome 2: $\mathrm{M}$ à Z)

Camille Hanlet, La Technique du style, Liège/Paris: Dessain, 1962

E.Legrand, Méthode de stylistique française à l'usage des élèves, Paris: Ed. J.de Gigord, 1986

Marcel Cressot, Le style et ses techniques, Paris: PUF, 1980

$\underline{\text { KOU BP } 2 / 2}$

Littré, Dictionnaire de la langue française, Tome 3, Monte Carlo: Editions du Cap, 1971

B et B. Lamizet, Dictionnaire orthographique, Paris: Editions Garnier, 1974

Robert Sctrick, Le Français aujourd'hui Ecrire, Parler: Les 100 difficultés du français, Paris: Presses Pocket, 1982

Maurice Grévisse, Savoir accorder le participe passé, Paris: Duculot, 1982

J.Blondé, P.Dumont et D.Gontier, Lexique du français du Sénégal, Dakar/Paris: NEA/ EDICEF, 1979

Marcel Cohen, Le subjonctif en français contemporain, Paris: SEDES, 1965

Jacqueline Picoche, Nouveau dictionnaire étymologique du français, (Usuels), Paris:

Hachette/Tchou, 1971

Maurice Grévisse, Le Bon Usage, Gembleux, Duculot, 1961

Etiemble et Jeanne Etiemble, L'Art d'écrire, Paris: Seghers, 1970

R.Galisson et D.Coste, Dictionnaire didactique des langues, Paris: Hachette, 1976

P.Gilbert, Dictionnaire des mots contemporains, (Usuels), Paris: Robert, 1980

Le nouveau Bescherelle, L'Art de conjuguer, Paris: Hatier, 1959

Henri Sensine, L'Emploi des temps en français, Paris: Editions Roudil, 1981

Henri Sensine, L'Emploi des temps en français, Paris: Payot, 1966

J-Y Dournon, Dictionnaire d'orthographie et des difficultés du français, Paris: Livre de poche, 1982

J-Y Dournon, Dictionnaire d'orthographie et des difficultés du français, Paris: Livre de poche, 1982

Claude Aziza et Robert Sctrick, Dictionnaire orthographique, Paris: Presses Pocket, 1982

J-P. Laurent, Rédiger pour convaincre, Paris: Duculot, 1984

Raymond Jacquenod, La Ponctuation maîtrisée, Alleur: Marabout, 1993 
Jacques Cellard, Le Subjonctif: Comment l'écrire? Comment l'employer?, Paris: Duculot, 1983

Louis Maynard, Dictionnaire des Lyonnaiseries Tome III, Lyon: Jean Honoré, 1982

$\underline{\text { KOU BP } 5}$

Maurice Grévisse, Le Bon Usage, Gembleux, Duculot, 1975

L. Duponchel, Dictionnaire du français de Côte-d'Ivoire, Abidjan: Institut de Linguistique Appliquée, 1975

Henri Bertaud du Chazaud, Nouveau dictionnaire des synonymes, Paris: Hachette/Tchou, 1971

Mariette Meunier Crespo, Des données empiriques à l'objet d'étude: La constitution d'un corpus de démodialectologie (français populaire d'Abidjan), Université Jean Moulin, Lyon III, Thèse en 2 volumes.

Antoine Albalat, La formation du style par l'assimilation des auteurs, Paris: Armand Colin [1901], 1999

Ferdinand de Saussure, Cours de Linguistique Générale, Paris: Payot, 1967

Pierre Guiraud, La Stylistique, 'Que Sais-je?', Paris: PUF, 1979

Frédéric Deloffre, La Phrase française, Paris: SEDES, 1979

Chantal Burdin, Le Français moderne tel qu'on le parle, Paris: Editions de Vecchi, 1981

P.Barucco, Eléments de Stylistique, Paris: Editions Roudil, 1972

Albert Dauzet, Tableau de la langue française, Paris: Payot [sans date]

Henri Benac, Guide alphabétique des difficultés du français, Paris: Hachette, 1978

Larousse, Dictionnaire des citations françaises, Paris: 1977

KOU BP6 B2 1/2

Littré, Dictionnaire de la langue française, Tomes 1, 2 et 4, Monte Carlo: Editions du Cap, 1971

Le Petit Larousse grand format, 1998

Jean Cauvin, Comprendre les Proverbes, Isy-les-Moulineaux: Ed St Paul, 1981

Grevisse, Le Français correct, Gembleux: Duculot, 1973

Charles Goedert, Guide pratique de grammaire française, Paris: Hachette, 1978

Marcel Lacarra, Les Temps des verbes, Gembleux: Duculot, 1984

J-P. Colignon et P-V. Berthier, La Pratique du style, Gembleux: Duculot, 1984

\section{NOTES}

1. Pierre-Marc de Biasi, Génétique des textes, Paris, CNRS Éditions, 2011, p. 261.

2. «Je n'ai pas fait de formation littéraire mais c'est la nécessité qui m'a amené à écrire. » Patrick Corcoran, « Entretien avec Ahmadou Kourouma », ASCALF Bulletin, n 24, 2002, p. 20.

3. Lamine Diabaté, homme politique, ami et codétenu de Kourouma. 
4. Documents déposés dans les archives de l'Institut Mémoire de l'édition contemporaine (IMEC) sous la cote KOU 1.

5. Patrick Corcoran, Daniel Delas et Jean-Francis Ekoungoun (éd.), Les Soleils des indépendances d'Ahmadou Kourouma: une longue genèse, Paris, CNRS Éditions, coll. «Planète Libre essais ", 2017, p. 150.

6. Patrick Corcoran, art. cit., p. 20.

7. Voir Florence Davaille, «La publication au Québec : l'énigme d'une rencontre inattendue », in Patrick Corcoran, Daniel Delas et Jean-Francis Ekoungoun (éd.), op.cit., p. 71-93.

8. Roland Barthes, «Écrivains et écrivants », in Essais critiques, Paris, Seuil, 1964, p. 148.

9. Ibid., p. 149.

10. Ahmadou Kourouma, Je témoigne pour l'Afrique, Grigny, Paroles de l'aube, 1998, p. 7.

11. Patrick Corcoran, art. cit., p. 22.

12. Daniel Delas, "Devenir écrivain: comment un écrivain «inculte» est devenu un écrivain » culte » ", in Patrick Corcoran, Daniel Delas and Jean-Francis Ekoungoun (éd.), op.cit., p. 239.

13. Ibid., p. 240.

14. Afrique littéraire et artistique, avril 1970.

15. Jean-Michel Djian, Ahmadou Kourouma, Paris, Seuil, 2010, p. 61-62.

16. Jeune Afrique Économie, $\mathrm{n}^{\circ} 139$, janvier 1991, p. 102-107. Propos recueillis par Odile Felgine et Michel Lobé Ewané.

17. Ahmadou Kourouma, cité dans Jean-Michel Djian, op.cit., p. 99.

18. Ahmadou Kourouma, "Pour une francophonie des peuples, des cultures et des dieux", Abidjan, 18 mars 1994.

19. Ahmadou Kourouma, Projet de préface, IMEC : KOU 24.

20. Michèle Zalessky, "La langue: un habit cousu pour qu'il moule bien » (entretien avec Ahmadou Kourouma), Diagonales, $n^{\circ} 7$, juillet 1988, supplément du Français dans le monde, $\mathrm{n}^{\circ} 218$, p. 4-5.

21. Ahmadou Kourouma, «Les dits et les non-dits », Wellesley College, Massachusetts, 1999.

22. La bibliothèque de... Ahmadou Kourouma, 14 novembre 2003, RFI (propos recueillis par Tirthankar Chanda).

23. Voir en appendice l'inventaire des dictionnaires et livres de référence de Kourouma.

24. Marcel Cressot, Le Style et ses techniques, Paris, PUF, 1980, p. 291-292.

25. Pierre Guiraud, La Stylistique, Paris, PUF, coll. « Que sais-je ? », 1979, p. 96.

26. Lettre à E. Gaède (faculté des Lettres de Nice), 31 octobre 1978. IMEC : KOU 22. Le roman auquel Kourouma travaille est Monnè, outrages et défis.

27. Lettre à Arlette Chemain, non datée (début 1977). IMEC : KOU 22. Le livre en question est Monnè, outrages et défis.

28. Lettre à E. Gaède, 31 octobre 1978. IMEC : KOU 22.

29. Les deux cahiers portent sur la couverture l'intitulé «Marché d'hyène ou de chat ». IMEC: KOU 6.

30. Ahmadou Kourouma, » Vachon, l'ami qui m'a fait », Études françaises, 31 (2), 1995, p. 15-16 (doi :10.7202/035973ar).

31. Ibid., p. 16. 


\section{RÉSUMÉS}

Toute tentative d'élucidation des moments-clés, supposés être déterminants, dans le devenir d'un écrivain risque de privilégier la biographie de l'auteur en question et de nous éloigner de l'étude analytique de son écriture. L'un des défis inhérents à la notion même d'une genèse auctoriale est celui de savoir comment transposer les méthodes et les outils employés par la génétique textuelle dans cet autre domaine, infiniment plus insaisissable, d'une vie humaine. Conscient de cette difficulté, cet article examine le parcours suivi par Ahmadou Kourouma vers la publication de son premier roman, Les Soleils des indépendances. Le contexte historique dans lequel ce projet d'écriture a vu le jour est esquissé, non pas pour expliquer l'existence du roman, mais pour confirmer l'une des sources majeures de la motivation de son auteur. L'histoire éditoriale du roman est également évoquée, mais sans lui conférer une valeur explicative quelconque. Refusant d'entériner l'opinion exprimée par Kourouma lui-même dans un court article sur le rôle joué par Georges-André Vachon dans la publication de son roman («Vachon, l'ami qui m'a fait »), l'article s'appuie sur une analyse du modus scribendi de Kourouma et sur une étude des multiples traces matérielles fournies par les archives pour dresser le portrait d'un écrivain, autodidacte certes, mais aussi acharné que méticuleux. Passionné par les dictionnaires et les livres de stylistique, Kourouma souhaitait vivement devenir écrivain et c'est grâce à une pratique scripturale menée sur de longues années, accompagnée d'un questionnement incessant sur sa propre pratique, qu'il a su réaliser ses ambitions.

\section{INDEX}

Mots-clés : Kourouma, littérature ivoirienne, littérature francophone, génétique textuelle, Les Soleils des indépendances

\section{AUTEUR}

\section{PATRICK CORCORAN}

Professeur émérite, University of Roehampton 